\title{
Non-Bullous Pemphigoid: A Yet-to-Be Defined Disease Entity
}

\author{
Hui Mei Cheng ${ }^{a}$ Hong Liang Tey ${ }^{a, b, c}$ \\ ${ }^{a}$ National Skin Centre, Singapore, Singapore; ${ }^{b}$ Lee Kong Chian School of Medicine, Nanyang Technological \\ University, Singapore, Singapore; ${ }^{C}$ Yong Loo Lin School of Medicine, National University of Singapore, \\ Singapore, Singapore
}

\section{Keywords}

Autoimmune bullous diseases - Bullous skin disease .

Pemphigoid

\section{Dear Editor,}

We read with interest the article by Moar et al. [1] in which differences between non-bullous pemphigoid (NBP) and bullous pemphigoid (BP) were studied. The authors found that compared to BP, NBP patients were more likely to have a history of myocardial infarction, diuretic use, and pruritus responding poorly to treatment.

The current diagnostic criteria for NBP remain to be better defined and this study highlighted such a challenge. Here, NBP patients were classified as those who had met the criteria for diagnosis of BP based on current European S2k guidelines [2], with consistent histology, direct/ indirect immunofluorescence or serologic findings, combined with a lack of blisters. However, patients who developed blisters 3 months from the onset of pruritus were also classified as NBP, creating an overlap of patients in the prodromal/non-bullous phase of $\mathrm{BP}$ and those with true NBP, thereby introducing misclassification bias. Since blisters are known to develop weeks to months after the onset of pruritus in the natural history of BP, we suggest that of the 24 patients diagnosed as NBP, 17 who developed blisters may be more accurately classified as BP, albeit with a delayed presentation of blisters. The remaining 7 patients who never developed skin blisters could be considered as having bona fide NBP. Although the authors' method of classification had been practised in previous studies, this ambiguity in classification greatly impedes further understanding of NBP. As an example, a patient with a 6-month history of urticarial rashes who develops blisters at the 6th month of illness may receive a diagnosis of NBP or BP, depending on whether the patient presents to the dermatologist at the $3 \mathrm{rd}$ or the 6 th month, respectively.

Our attention was also drawn to the large number of patients who received a diagnosis of BP at the authors' institution but were excluded from the study. Of 532 patients, who received a diagnosis of BP, only 122 had satisfied criteria for BP. In other words, a large majority of patients who were diagnosed as BP have incomplete findings on clinical features, histology, and direct and indirect immunofluorescent and serologic investigations. Since a proportion of patients ultimately included in the study were without blisters, the basis on which a diagnosis of BP was made in the original population needs to be discussed as this may result in sampling bias and undermine its external validity. Specifically, we would like to caution against relying on enzyme-linked immunosorbent assays of BP180 and BP230 antibodies as a main criterion in the diagnosis of BP. BP180 and 
BP230 are auto-antibodies to basement membrane proteins which are commonly detected in normal subjects, especially in the older age groups, in which they do not exhibit binding to the NC16A domain and are not pathogenic, unlike in BP [3]. Even in the clinical context of itch, the presence of these antibodies does not necessarily equate to a diagnosis of BP. "Epitope spreading" is a phenomenon whereby a primary inflammatory process causes the release or exposure of a previously "hidden" endogenous epitope, leading to a secondary autoimmune response [4]. Postulated inciting events include trauma, such as scratching or rubbing, or inflammatory skin diseases, such as lichen planus, have been implicated in the pathogenesis of pemphigoid nodularis and lichen planus pemphigoides, respectively $[4,5]$. In other words, scratching/rubbing in response to pruritus, regardless of cause, can result in the disruption of the basement membrane and potentially the production of antibodies against self-antigens in the skin, especially BP180 antibodies. In addition, whether such secondary antibodies result in pathology is unclear. It has been shown that a proportion of patients with positive direct immunofluorescence did not eventually develop any autoimmune blistering disorder after a mean follow-up of 6 years [6]. Hence, to fulfill the diagnosis of BP, the interpretation of examination and investigation findings in an appropriate clinical context is imperative, particularly in patients presenting with itch without blisters.

With the article's concluding recommendation that NBP "should be considered as a cause of severe pruritus in the elderly," we would like to raise a note of caution against the overdiagnosis of NBP. In elderly patients presenting with severe pruritus, the presence of autoimmune antibodies may not be causative, particularly in the absence of blisters clinically. The observation of a blister in histology, especially one without inflammatory infiltration, may be consequent to rubbing. In addition, treatment targeted to inhibit antibody production, namely rituximab, has not been reported to be effective in the treatment of chronic pruritus. As the natural history of $\mathrm{BP}$ is that it remits within months to years, cases of nonblistering chronic itch for extended durations, such as decades in many cases of chronic pruritus, are unlikely to be attributable to NBP.

The disease entity of NBP, along with its diagnostic criteria and pathogenesis, needs to be better defined. After all, the Greek origin of "pemphigoid" is "form of a blister" (pemphix, blister; and eidos, form), which imparts an oxymoronic counter-intuition to the term and nosology of NBP. In patients presenting with chronic itch, NBP should not be overdiagnosed pending a better definition of this entity, as such a diagnosis would implicate routine institution of treatments for BP and may detract physicians and researchers from the actual pathogenesis and treatment of chronic pruritus.

\section{Key Message}

Non-bullous pemphigoid is a poorly defined disease entity; overdiagnosis in chronic pruritus should be avoided.

\section{Conflict of Interest Statement}

The authors have no conflicts of interest to declare.

\section{Funding Sources}

H.L.T. is supported by the Clinician Scientist Awards (NMRC/ CSA-INV/0023/2017 and CSAINV20nov-0003) conferred by Singapore's National Medical Research Council.

\section{Author Contributions}

H.L.T.: conceptualization; H.M.C.: manuscript preparation; H.M.C., H.L.T.: manuscript review and editing.

\section{References}

1 Moar A, Azzolini A, Tessari G, Schena D, Girolomoni G. Non-bullous pemphigoid: A single-center retrospective study. Dermatology. 2021:1-7.

2 Schmidt E, Goebeler M, Hertl M, Sárdy M, Sitaru C, Eming R, et al. S2k guideline for the diagnosis of pemphigus vulgaris/foliaceus and bullous pemphigoid. J Dtsch Dermatol Ges. 2015;13(7):713-27.

Non-Bullous Pemphigoid: Yet-to-Be Defined
3 Desai N, Allen J, Ali I, Venning V, Wojnarowska F. Autoantibodies to basement membrane proteins BP180 and BP230 are commonly detected in normal subjects by immunoblotting. Australas J Dermatol. 2008; 49(3):137-41.

4 Chan LS, Vanderlugt CJ, Hashimoto T, Nishikawa T, Zone JJ, Black MM, et al. Epitope spreading: Lessons from autoimmune skin diseases. J Invest Dermatol. 1998;110(2):103-9.
5 Powell A, Albert S, Gratian M, Bittencourt R, Bhogal B, Black M. Pemphigoid nodularis (non-bullous): a clinicopathological study of five cases. Br J Dermatol. 2002;147(2):343-9.

6 Lipsker D, Borradori L. 'Bullous' pemphigoid: what are you? Urgent need of definitions and diagnostic criteria. Dermatology. 2010; 221(2):131-4. 